\title{
Creencias sobre la insuficiencia renal crónica entre expertos y profanos
}

\section{Esperanza Vélez Vélez}

Doctora, Máster en Ciencias de la Enfermería, Máster en Docencia Universitaria, Profesora de la Escuela de Enfermería Fundación Jiménez Díaz-Idcsalud- Universidad Autónoma de Madrid

\section{Resumen}

Objetivo: Identificar las creencias y representaciones sobre la insuficiencia renal crónica (IRC) en pacientes, población general y expertos.

\section{Metodología:}

Estudio descriptivo transversal que analiza una muestra de 120 participantes seleccionados por muestreo no probabilístico por cuotas. Se crearon cinco grupos de estudio en función de su nivel de interacción con la enfermedad. La herramienta utilizada fue el Cuestionario de Creencias Sobre la Enfermedad.

\section{Resultados:}

Identidad, Controlabilidad, Mutabilidad, Responsabilidad Personal y Azar no presentan diferencias significativas entre los grupos estudiados. El factor Causas muestra diferencias estadísticamente significativas entre el grupo de pacientes con universitarios de ciencias de la salud y expertos $(p<0,001)$ $y$ entre otros universitarios con universitarios ciencias de la salud y expertos $(p<0.001)$. El factor Incapacidad muestra diferencias significativas en-

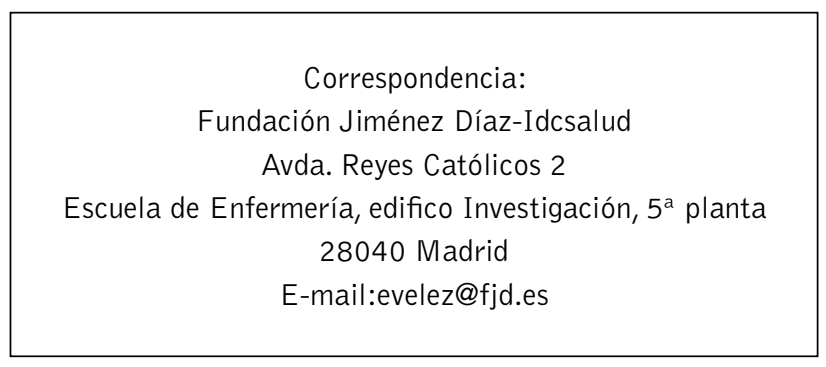

tre pacientes, universitarios y población general $(p<0,001)$ y entre expertos con otros universitarios y población general $(p<0,001)$. Los pacientes ofrecen un menor número de síntomas asociados tanto a la IRC como a la hemodiálisis (HD). Y en cuanto a las causas, las de origen biológico y conductuales son las más consideradas por todos los grupos. Solo el grupo de pacientes en HD considera el azar factor causal de la enfermedad.

\section{Conclusiones:}

En oposición al modelo científico, pacientes y población general comparten la idea de que la IRC es curable, depende en gran medida del azar y de hábitos de conducta y es menos grave de lo que opinan expertos. La experiencia con la enfermedad aporta más información (no necesariamente conocimiento) que es recordada en función de la utilidad para lograr un mayor bienestar o una mejora de los cuidados. 
Beliefs about chronic renal failure of experts and laypeople

\section{Abstract}

Objective: to identify beliefs and representations that patients, general public and experts keep about CKD.

\section{Methods:}

Cross-sectional study with a sample of 120 participants selected by non-probabilistic sampling. Five study groups were created according to their level of interaction with the disease. The tool used to identify and analyze the beliefs of the participants was the "Cuestionario de Creencias Sobre la Enfermedad" (Beliefs Questionnaire about Disease).

\section{Results:}

Identity, Controllability, mutability, Personal Responsibility and Chance present no significant differences among groups. Causes factor shows statistically significant difference between the group of patients with health science students and experts ( $p$ $<0.001$ ), and between health science students with students different from health sciences and experts $(p<0.001)$. Inability factor shows significant differences between patients, students different from health sciences and general population $(p<0.001)$ and between experts, general population and students different from health sciences $(p<0.001)$. Patients provide a smaller number of symptoms associated with both CKD and HD. As for the causes, biological and behavioral origin is the most considered by all groups. Only HD patients considered chance a possible causal factor of the disease.

\section{Conclusions:}

As opposed to what scientific models defend, patients and general population share the idea that chronic renal disease is a curable condition, that the onset depends mainly on chance and behavioral patterns, and that it is a less serious condition than what experts in renal care state. Suffering the disease offers more information about it (not necessarily knowledge) and this information is recalled according to its usefulness to achieve greater wellbeing or improvement in health care.

\section{KEYWORDS:}

- BELIEFS ABOUT THE DISEASE

- CHRONIC KIDNEY DISEASE

- HEMODIALYSIS, LAY AND EXPERT ILLNESS PERCEPTIONS

\section{Introducción}

El cuidado integral de los pacientes con IRC precisa que ésta sea entendida no sólo como lesión o daño orgánico sino como la forma en la que el individuo, dadas sus características psíquicas y socioculturales, sufre o padece la enfermedad.

Una manera de abordar la enfermedad teniendo en cuenta la perspectiva personal y sociocultural de la misma es considerando las representaciones o creencias que los sujetos mantienen respecto de la misma.

El interés de la investigación de representaciones de la enfermedad estriba en la aplicación de los resultados a tres campos: el autodiagnóstico, búsqueda sanitaria y toma de decisiones sobre cuidado; cumplimiento y adherencia al tratamiento; y reacciones de la población ante las víctimas de la enfermedad. Conocer las diferencias entre las creencias que mantienen los pacientes sobre su enfermedad y las mantenidas por los expertos puede ser de utilidad en el desarrollo de materiales educativos.

Se ha elegido el modelo de las representaciones de la enfermedad como marco teórico y conceptual porque es un método cuantitativo ampliamente utilizado en la investigación de las percepciones y creencias de la enfermedad, existen herramientas psicométricas fiables y validadas $^{1,2}$ y se ha utilizado para comparar las representaciones de pacientes y las de cuidadores y población general o profanos. Este último punto es importante cuando se quieren hacer predicciones sobre como expertos y profanos difieren en términos de representaciones de la enfermedad.

En general, se comparte la idea de que los pacientes tienen representaciones similares a las de los expertos, y de esta hipótesis se parte en este trabajo; por ejemplo, se cree que los pacientes tienen un buen conocimiento de la 
enfermedad por su experiencia con ella, y que sus cuidadores comparten ideas más cercanas a las de la población general o los profanos. No obstante no se cuenta con estudios suficientes para desarrollar hipótesis más detalladas ${ }^{3}$.

Aunque son muchos los estudios sobre las representaciones de la enfermedad y su impacto en la respuesta del paciente (conductual y emocional) ${ }^{1}$, son escasos los estudios sobre las representaciones de la enfermedad desde la perspectiva de los familiares o cuidadores. Los estudios al respecto indican que las representaciones de familiares y cuidadores influyen las respuestas de los pacientes a su enfermedad ${ }^{4,5}$. Heijmans et al. demostraron que la pareja tiene una visión más negativa de la enfermedad que el propio paciente ${ }^{5}$. Éste ve su enfermedad con mayor posibilidad de cura, con menos consecuencias y de menor duración. Las discrepancias entre las percepciones de los pacientes y sus cuidadores tuvieron un impacto negativo sobre la adaptación del paciente a su enfermedad.

El estudio de las diferencias entre las representaciones de la enfermedad de expertos y profanos permite una mejor comprensión sobre cómo desarrollar intervenciones educacionales y estrategias de comunicación con los pacientes ${ }^{6}$. Así, Ferguson et al. compararon niveles de conocimiento, percepciones de riesgo y confianza en las fuentes de información en grupos de expertos y profanos en el caso concreto de las transfusiones de sangre. Los autores argumentan que es necesario analizar las creencias de expertos y profanos, ya que estos grupos son la parte interesada cuando se negocian las opciones de tratamiento. En el estudio de Ferguson et al., estos grupos discreparon con respecto al tipo de información que consideraron "de confianza". Así, para los expertos las fuentes más fiables de información son las científicas, mientras que los profanos tienden a depositar su confianza en fuentes populares (amigos, periódicos y otros medios de divulgación) ${ }^{6}$.

El conocimiento de las discrepancias en las representaciones entre expertos y profanos es importante, especialmente para los expertos, en la mejora de las estrategias de comunicación entre ambos grupos. Este conocimiento permite mejorar la confianza e identificar cuándo los riesgos de comunicación de las distintas fuentes pueden ser más o menos efectivos.

Marco conceptual: Modelo de las Representaciones de las Enfermedad: Las representaciones sobre un padecimiento crónico comprenden no sólo información sobre las concepciones colectivas de lo que constituye el enfermar, padecer y atender una enfermedad, sino también sobre cómo estos procesos fueron construidos y reelaborados a la luz de las experiencias personales y las colectivas, ya sea con otros enfermos, familiares, profesionales de la salud e instituciones.

La escuela que ha profundizado en el estudio de las representaciones sociales es la francesa; sin embargo, frente a la propuesta europea, iniciada por Moscovici y secundada por varios autores entre quienes destaca Jodelet, mucho más teórica, surge una corriente americana, mucho más práctica - Leventhal et al. como pioneros-, una corriente distinta no sólo por utilizar otros mecanismos de medición a los utilizados por la corriente europea, sino también por abrir nuevos caminos de aplicación, en concreto todo el campo de estudio de la representación de la enfermedad ${ }^{7}$.

Leventhal construyó un Modelo de Sentido Común de la enfermedad (Common Sense Model) el cual es un modelo de autorregulación que propone que el paciente construye activamente una definición o representación de la enfermedad y regula la conducta en base a esta representación. La teoría se basa en observaciones cuantitativas y cualitativas que reflejan los patrones de conducta del paciente. El sistema de base se compone de una serie e etapas que guían la acción adaptativa ${ }^{8}$.

El concepto de representación incluye síntomas y creencias. Los síntomas preceden en realidad a la representación de la enfermedad y activan la respuesta del paciente. Estas reacciones pueden ser muy variables. En ocasiones síntomas triviales son tenidos en cuenta mientras síntomas más serios son ignorados. Esta respuesta variable está influida por la cultura y los subgrupos. La actitud del rol de enfermo difiere entre grupos sociales/ culturales y conduce a diferentes tasas de información o registro de enfermedades. La percepción de los síntomas está influida por varios factores incluyendo la frecuencia de los síntomas, su familiaridad, la predicción de los resultados, la amenaza de la enfermedad, y las pérdidas potenciales de la enfermedad. Sólo los síntomas parecen estimular las creencias sobre la enfermedad. Si un síntoma se interpreta como un signo definitivo de enfermedad, entonces se buscará el diagnóstico y el tratamiento.

Revisión de la literatura: En general, los estudios previos muestran que la representación de la enfermedad está muy relacionada a la adherencia al tratamiento y recomendaciones médicas, y es también consecuencia del modelo específico de la enfermedad desarrollado 
por los pacientes, el cual difiere con relativa frecuencia de las explicaciones médicas?.

En concreto, la representación de la enfermedad renal para el sujeto que la padece es un área poco explorada comparada con otras enfermedades como la diabetes, el cáncer o el sida, y los pocos estudios existentes se centran en el contenido específico de la representación.

Polaschek ${ }^{10}$, Parfeniil ${ }^{11}$ y Lin ${ }^{12}$, confirman la escasez de estudios tanto cualitativos como cuantitativos sobre las representaciones de la IRC. Más escasos son los estudios que analizan las percepciones de la enfermedad que mantienen las familias de los pacientes, y nulos los que analizan las representaciones de la IRC de la población general o de aquellos con poco o ningún contacto con la enfermedad.

El análisis de las representaciones de la enfermedad que mantienen expertos y profanos constituye una estrategia que intenta conciliar y acercar el conocimiento de la enfermedad que tienen los que la padecen y aquellos que los cuidan. Este es un abordaje necesario si queremos profundizar en la estructura del conocimiento, y el primer paso en la elaboración de intervenciones educativas con garantías de éxito. No olvidemos que las representaciones dirigen la atención hacia la información y sirven de guía en la selección de estrategias de afrontamiento.

Desde el marco teórico de las representaciones sociales y los modelos explicativos de Kleinman, y con una metodología cualitativa narrativa, un estudio previo de la autora ${ }^{13}$, que analiza los significados de la enfermedad en un grupo de pacientes en HD, destaca dos hallazgos que guían esta investigación: la fuerte influencia del contexto y del grupo en el que socializan como origen de las representaciones y el escaso impacto de la consulta prediálisis; un porcentaje importante de los pacientes entrevistados no recuerda haber recibido información previa sobre la enfermedad y la diálisis antes de su entrada en programa, aun cuando la mayoría tenían registrada en su historia clínica el paso por la consulta prediálisis. Las autoras concluyen que no basta con que al paciente se le ofrezca información científica considerada necesaria para el buen manejo de la enfermedad; se debe trabajar con mayor énfasis en la cuestión de la distancia con la cual se percibe esta información.

\section{Objetivos}

Objetivo general: Identificar las representaciones 0 creencias sobre la IRC en diferentes grupos con distinto grado de experiencia en la enfermedad.

\section{Objetivos específicos:}

- Identificar las representaciones de la IRC en los distintos dominios de la enfermedad entre los grupos de estudio.

- Analizar y contrastar las diferencias entre profanos y expertos.

\section{Material y métodos}

Diseño: Estudio descriptivo transversal que utiliza como herramienta el cuestionario.

Ámbito y sujetos de estudio: El estudio se ha realizado con pacientes, profesionales y estudiantes de Enfermería de la Fundación Jiménez Díaz - idcsalud(FJD), con estudiantes de Administración y Dirección de Empresas (ADE) de la Universidad Carlos III y con población general.

En función de su proximidad con la enfermedad, los grupos de estudio quedaron así configurados: cuatro grupos con una relación más cercana con la IRC:

1. Pacientes con una IRC en hemodiálisis (Ptes HD), con su experiencia con la enfermedad y con el tratamiento con hemodiálisis.

2. Pacientes IRC en prediálisis (Ptes prediálisis): experiencia con la enfermedad pero sin experiencia en el tratamiento con diálisis.

3. Enfermeras de cuidados generales y estudiantes de último año de Enfermería (Univ. CCSS y Enf. gral): expertos en conocimiento científico médico general.

4. Expertos: enfermeras y médicos de la unidad de diálisis (Expertos): expertos en conocimiento médico específico de la enfermedad.

$Y$ dos grupos a los que por su distancia con la IRC consideraremos profanos o novatos:

5. Universitarios no relacionados con las CCSS, en concreto estudiantes de ADE (Univ. No CCSS).

6. Sujetos no universitarios o que hace tiempo que terminaron los estudios (Población general).

Características de la muestra: Por muestreo no probabilístico por cuotas, se seleccionaron aproximadamente 20 participantes para cada uno de los grupos que 
conforman la muestra total del estudio. Finalmente, la muestra quedó configurada por un total de 120 participantes repartidos según se recoge en el gráfico 1 . La edad y sus datos de dispersión quedan reflejados en el gráfico 2.

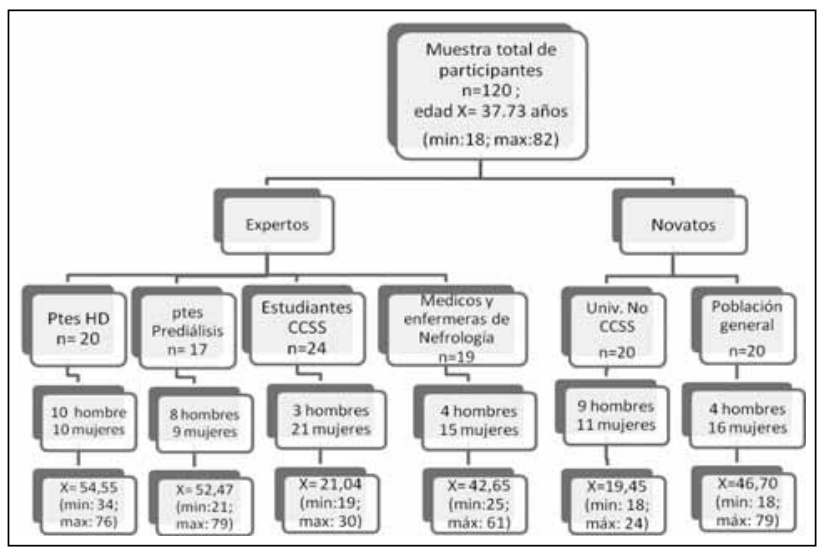

Gráfico 1. Grupos participantes en el estudio.

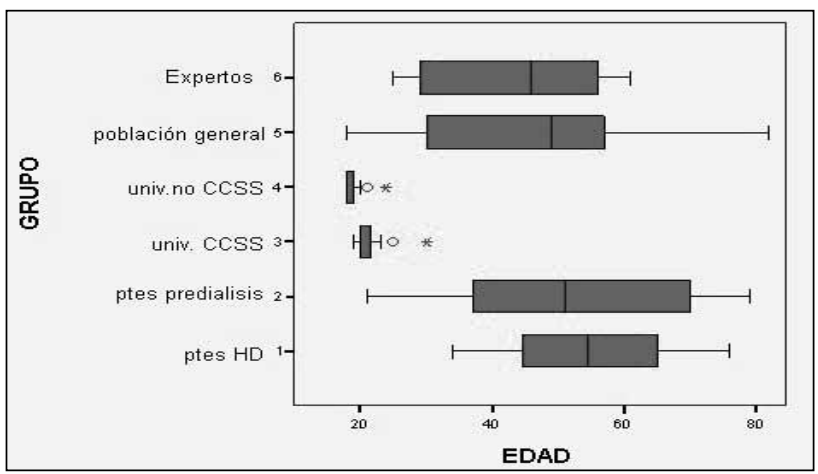

Gráfico 2. Mediana y valores de dispersión de la edad de la muestra.

Herramienta: Para identificar y analizar las representaciones de la IRC de los participantes se utilizó el Cuestionario de Creencias Sobre la Enfermedad (CCSE) de Van-Der-Hofstadt y Rodríguez Marín ${ }^{14}$, adaptado para este estudio.

EI CCSE consta de tres apartados: El primero de ellos contiene 27 afirmaciones sobre la IRC, agrupadas en factores: Identidad (características y declaraciones definitorias sobre la enfermedad y sus síntomas), Causa (posibles causas, ofrece causas inconcretas de la enfermedad frente a concretas, a mayor puntuación mayor concreción), Incapacidad (incapacidad durante la enfermedad, consecuencias de la misma), Cura (posibilidades de cura), Responsabilidad personal (influencia de hábitos sobre el origen de la enfermedad), Controlabilidad (posibilidad de control de la enfermedad), Mutabilidad (estabilidad de la enfermedad y sus síntomas a lo largo de la duración) y Azar (influencia de la casualidad o la suerte), para las que los participantes determinaron su nivel de acuerdo mediante una escala de likert de 5 puntos (desde 1 para "muy en desacuerdo" hasta 5 para "muy de acuerdo"), escala que se redujo a 3 puntos para facilitar el análisis de los resultados, la escala de valoración quedó convertida en una escala de tres puntos ( $1=$ En desacuerdo, $2=$ No sé, $3=$ De acuerdo).

El segundo apartado del cuestionario incluye una lista de 18 síntomas que los participantes asignan a la IRC y/o la HD con opciones de respuesta "si", "no", y que nos informan de aquellos síntomas que los participantes asocian con mayor frecuencia a la IRC y cuales asocian con mayor frecuencia a su tratamiento, la hemodiálisis.

El último apartado pretende precisar las causas de la IRC. Incluye una lista de 18 posibles causas que son valoradas por los participantes utilizando la escala de 5 puntos.

Procedimiento: Los seis grupos que constituyen la muestra cumplimentaron el CCSE. El cuestionario, de autocumplimentación, se recogía el mismo día que se entregaba o en un plazo de 48 a 72 horas.

El reclutamiento de participantes, la distribución y recogida del cuestionario entre los grupos participantes se realizó durante los meses de octubre-diciembre, y el tiempo dedicado a su cumplimentación osciló entre 10 y 30 minutos.

Consideraciones éticas: La participación en el estudio no supuso ningún riesgo ni beneficio para los participantes. Se obtuvo permiso para realizar el estudio de los directores médicos y supervisores de las unidades de diálisis de donde fueron reclutados los participantes. También se obtuvo la aprobación del Comité Ético de Investigación Clínica de la Fundación Jiménez Díazidcsalud.

Los potenciales participantes en el estudio recibieron una carta de la investigadora que contenía la información de un consentimiento informado estándar de investigación. Para preservar la confidencialidad de los participantes, en la carta se les explicaba que la cumplimentación de los cuestionarios supondría su consentimiento a participar en el estudio, evitando con ello la firma del consentimiento informado, único dato que podría identificarles. La carta ex- 
plicaba también que la información de los cuestionarios se trataría con rigurosa confidencialidad, en cumplimiento con lo establecido por la Ley Orgánica 15/1999, de 13 de diciembre. Los cuestionarios se marcaron con un código y la identidad de los participantes ha permanecido anónima.

Análisis de los datos: Para el análisis de los datos se utilizó el software estadístico SPSS 15.

Se realizó un análisis descriptivo de los 8 factores 0 dominios en los que se agrupan las 27 variables del cuestionario y también se calculó la normalidad y la homogeneidad de las variables. Aunque los datos del test de normalidad no permiten rechazar la distribución normal de los datos, la disparidad de varianzas en tres de los factores y el tamaño de la muestra hace que nos decantemos por medidas no paramétricas para el análisis de los resultados. Así, el contraste entre grupos - contraste de medias- se realizó aplicando las pruebas no paramétricas de Kruskal Wallis y $U$ de Mann Whitney. Con el test estadístico Kruskal Wallis se estableció si las variables analizadas diferían o no entre los distintos grupos. Con el test $U$ Mann Whitney se analizó entre qué grupos se encontraba la diferencia mediante comparaciones 2 a 2 (6 grupos). Se aplicó la corrección de Bonferroni para controlar la posibilidad de cometer errores de tipo I, considerando estadísticamente significativos valores $p \leq 0,003$.

También analizamos la magnitud de la diferencia de medias, calculando el tamaño del efecto mediante el estadístico $d$ de Cohen.

Además del análisis de los factores, se realizó un análisis detallado de cada variable o ítem que conforma cada factor. Para este análisis, la escala de valoración se agrupo en tres puntos ( $1=$ En desacuerdo, $2=$ No sé, $3=$ De acuerdo) y se analizó el contraste de porcentajes mediante la prueba estadística Ji cuadrado o el test exacto de Fisher - de los que se ofrece el valor de $p$, considerando significativos valores $p<0,05$ - cuando el tamaño de la muestra y el número de frecuencias esperadas no permitió utilizar el primero.

\section{Resultados}

\section{Creencias sobre la enfermedad (apartado 1 del CCSE)}

La tabla 1 recoge las puntuaciones medias que obtienen los distintos grupos en las 8 dimensiones de la enfermedad analizadas. Los factores Identidad, Controlabilidad, Mutabilidad, Responsabilidad Personal y Azar no presentan diferencias significativas entre los distintos grupos $(p>0.05)$.

Del análisis estadístico de los factores en los que se encontró diferencia entre grupos (test $U$ de Mann Whitney (comparaciones dos a dos)) cabe destacar:

- En el factor cura, universitarios y expertos ofrecen la puntuación más baja (menor posibilidad de cura) y los pacientes prediálisis y grupos con poca experiencia con la enfermedad las puntuaciones más alta. Se observan diferencias estadísticamente significativas entre el grupo de pacientes prediálisis, universitarios de CCSS y expertos $(p<0.002 ; d=1,26)$ y $d=$ 1,27 , respectivamente), y entre universitarios N0 CCSS con universitarios CCSS y expertos $(p<0,001$; $d=2,12$ y $d=2.15$ ).

- En el factor Causas, pacientes tanto en HD como en prediálisis, ofrecen mayor concreción que el grupo de expertos en este factor (media 2,59 vs 1,38 ).

\begin{tabular}{|c|c|c|c|c|c|c|c|}
\hline & $\begin{array}{c}\text { Ptes } \\
\text { HD }\end{array}$ & $\begin{array}{l}\text { Ptes pre- } \\
\text { diálisis }\end{array}$ & $\begin{array}{c}\text { Univ. } \\
\text { CCSS y } \\
\text { enf. gral }\end{array}$ & $\begin{array}{l}\text { Univ. } \\
\text { no } \\
\text { CCSS }\end{array}$ & $\begin{array}{c}\text { Población } \\
\text { general }\end{array}$ & Expertos & \\
\hline & $\begin{array}{c}\text { Media } \\
\text { (DT) }\end{array}$ & $\begin{array}{c}\text { Media } \\
\text { (DT) }\end{array}$ & $\begin{array}{l}\text { Media } \\
\text { (DT) }\end{array}$ & $\begin{array}{c}\text { Media } \\
\text { (DT) }\end{array}$ & $\begin{array}{c}\text { Media } \\
\text { (DT) }\end{array}$ & $\begin{array}{c}\text { Media } \\
\text { (DT) }\end{array}$ & $\mathbf{P}_{\mathrm{KW}}$ \\
\hline Identidad & $\begin{array}{c}3,03 \\
(0,53)\end{array}$ & $\begin{array}{c}3,02 \\
(0,64)\end{array}$ & $\begin{array}{c}2,30 \\
(0,35)\end{array}$ & $\begin{array}{c}3,20 \\
(0,49)\end{array}$ & $\begin{array}{c}3,17 \\
(0,67)\end{array}$ & $\begin{array}{c}3,34 \\
(1,90)\end{array}$ & 0,240 \\
\hline Cura & $\begin{array}{c}2,63 \\
(0,75)\end{array}$ & $\begin{array}{c}2,70 \\
(0,83)\end{array}$ & $\begin{array}{c}1,90 \\
(0,33)\end{array}$ & $\begin{array}{c}2,74 \\
(0,45)\end{array}$ & $\begin{array}{c}2,51 \\
(0,83)\end{array}$ & $\begin{array}{c}1,90 \\
(0,32)\end{array}$ & $<0,001$ \\
\hline Causa & $\begin{array}{c}2,59 \\
(1,12)\end{array}$ & $\begin{array}{c}2,38 \\
(0,91)\end{array}$ & $\begin{array}{c}1,48 \\
(0,55)\end{array}$ & $\begin{array}{c}2,34 \\
(0,74)\end{array}$ & $\begin{array}{c}2,33 \\
(1,06)\end{array}$ & $\begin{array}{c}1,38 \\
(0,43)\end{array}$ & $<0,001$ \\
\hline Mutabilidad & $\begin{array}{r}3,38 \\
(1,04)\end{array}$ & $\begin{array}{c}3,00 \\
(0,77)\end{array}$ & $\begin{array}{c}3,38 \\
(0,52)\end{array}$ & $\begin{array}{c}3,43 \\
(0,54)\end{array}$ & $\begin{array}{c}3,6 \\
(0,62)\end{array}$ & $\begin{array}{c}3 \\
(0,47)\end{array}$ & 0,022 \\
\hline Incapacidad & $\begin{array}{c}3,28 \\
(0,86)\end{array}$ & $\begin{array}{c}3,18 \\
(0,78)\end{array}$ & $\begin{array}{c}3,83 \\
(0,70)\end{array}$ & $\begin{array}{c}4,07 \\
(0,56)\end{array}$ & $\begin{array}{c}4,2 \\
(0,49)\end{array}$ & $\begin{array}{c}3,37 \\
(0,60)\end{array}$ & $<0,001$ \\
\hline Controlabilidad & $\begin{array}{c}3,10 \\
(1,01)\end{array}$ & $\begin{array}{c}2,71 \\
(0,81)\end{array}$ & $\begin{array}{c}3,17 \\
(0,95)\end{array}$ & $\begin{array}{c}3,43 \\
(0,78)\end{array}$ & $\begin{array}{c}3,05 \\
(0,96)\end{array}$ & $\begin{array}{l}3,26 \\
(1,2)\end{array}$ & 0,094 \\
\hline $\begin{array}{l}\text { Responsabilidad } \\
\text { personal }\end{array}$ & $\begin{array}{c}3,4 \\
(0,91)\end{array}$ & $\begin{array}{c}3,32 \\
(0,66)\end{array}$ & $\begin{array}{c}3,31 \\
(0,92)\end{array}$ & $\begin{array}{c}3,25 \\
(0,73)\end{array}$ & $\begin{array}{c}3,3 \\
(0,78)\end{array}$ & $\begin{array}{c}3,61 \\
(0,67)\end{array}$ & 0,668 \\
\hline Azar & $\begin{array}{c}2,95 \\
(1,73)\end{array}$ & $\begin{array}{c}2,71 \\
(1,40)\end{array}$ & $\begin{array}{c}1,75 \\
(1,07)\end{array}$ & $\begin{array}{c}2,60 \\
(1,14)\end{array}$ & $\begin{array}{l}2,45 \\
(1,5)\end{array}$ & $\begin{array}{l}1,82 \\
(1,1)\end{array}$ & 0,040 \\
\hline
\end{tabular}

Tabla 1. Medias y desviaciones estándar de las variables estudiadas en los distintos grupos que constituyen la muestra. Nivel crítico establecido $p \leq 0,05$. 
Las diferencias son estadísticamente significativas entre pacientes $H D$, universitarios de CCSS y expertos ( $p<0,001 ; d=1.25$ y $d=1.42$, respectivamente), entre pacientes prediálisis, universitarios CCSS y expertos $(p<0,001 ; d=1.19$ y $d=1.40$, respectivamente), y entre universitarios NO CCSS, universitarios CCSS y expertos ( $p<0.001 ; d=1,31$ y $d=1.58$, respectivamente).

- En el factor Incapacidad, la puntuación más alta (enfermedad más incapacitante) la presenta el grupo de población general, y la más baja el de paciente prediálisis. Se encuentran diferencias significativas entre pacientes HD con universitarios NO CCSS y población general $(p<0,001 ; d=0.95 \mathrm{y} d=-1.31$, respectivamente), pacientes prediálisis y universitarios No CCSS y población general $(p<0,001 ; d=-1,31$ y $d=-1,56$, respectivamente), y expertos con universitarios No CCSS y población general $(p<0,001, d=$ 1,20 y d=1,51).

Los gráficos 3 y 4 recogen el análisis detallado de aquellos ítem de los factores cura e incapacidad que presen-

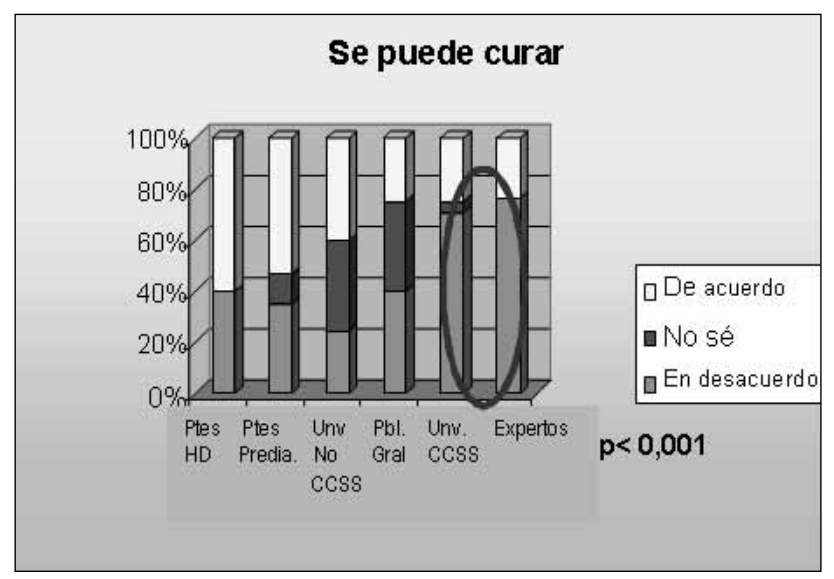

Gráfico 3.

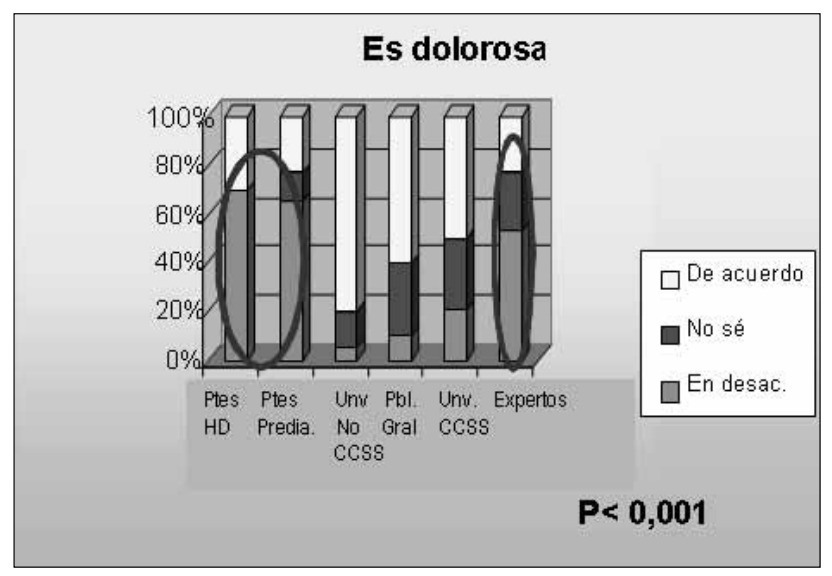

Gráfico 4. tan mayor discrepancia entre grupos, corroborando los hallazgos del análisis de los factores. En el gráfico 3, el grupo de pacientes HD y prediálisis ofrecen un porcentaje mayor y significativamente distinto al resto de los grupos sobre la posibilidad de cura de la IRC (60 y $52,9 \%$, respectivamente).

Respecto a la incapacidad, el ítem de este factor que muestra mayor discrepancia entre grupos es "la IRC es dolorosa" (gráfico 4); pacientes y expertos nefrológicos, con un contacto más directo con la enfermedad, comparten una idea similar entre sí, pero diferente a la mantenida por el resto de los grupos.

\section{Síntomas asociados a IRC y a HD (apartado 2 del CCSE)}

Dada la similitud de respuestas entre pacientes HD y prediálisis por un lado, universitarios CCSS y expertos por otro y universitarios no CCSS y población general

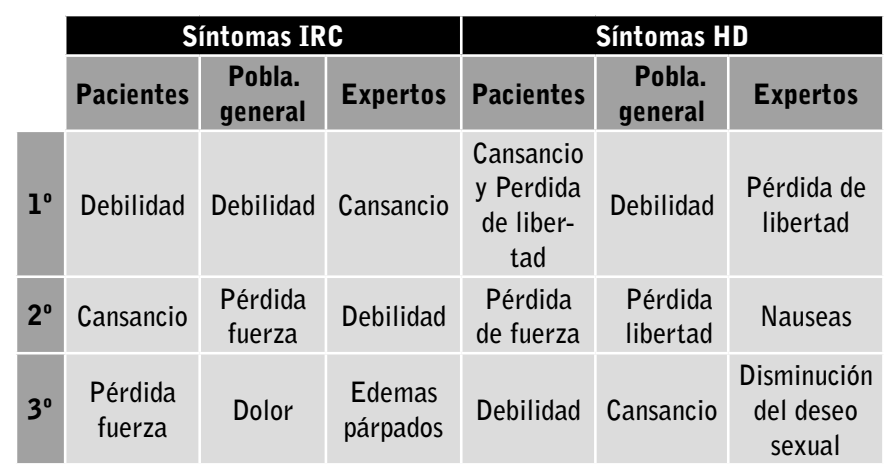

Tabla 2. Síntomas más asociados a la IRC y a la HD por los grupos estudiados.

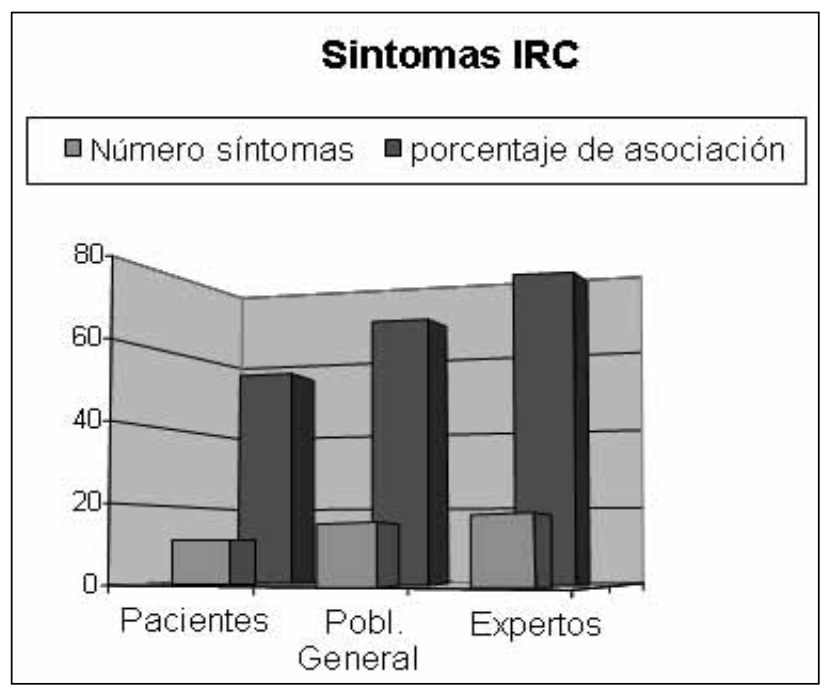

Gráfico 5. Número de síntoma que asocia cada grupo a la IRC, y porcentaje de asociación. 
por otro, se agrupó la muestra de estudio en estos tres grupos: pacientes, población general y expertos.

Los pacientes ofrecen un menor número síntomas asociados a la IRC y el porcentaje de asociación también es menor que el ofrecido por la población general y el grupo de expertos. En cuanto a los síntomas asociados a la HD, el patrón es similar (gráficos 5 y 6). La tabla 2 recoge los tres síntomas asociados con mayor frecuencia a la IRC y a la HD, según los grupos analizados.

\section{Causas (apartado 3 CCSE)}

Para su análisis, las 18 posibles causas ofrecidas como posible factor etiológico de la IRC se agruparon en $4 \mathrm{ca}$ tegorías: Causas Biológicas (herencia, envejecimiento, alteración del sistema inmune, gérmenes o virus), Psicosociales (actitud mental muy negativa, estado emocional propicio, personalidad, estrés o preocupaciones, problemas familiares), Conductuales y/o ambientales (escaso cuidado médico en el pasado, hábitos poco adecuados, consumo de alcohol, consumo de tabaco, dieta y hábitos alimenticios, polución, accidente o trauma físico, exceso de trabajo) y Azar (mala suerte o destino).

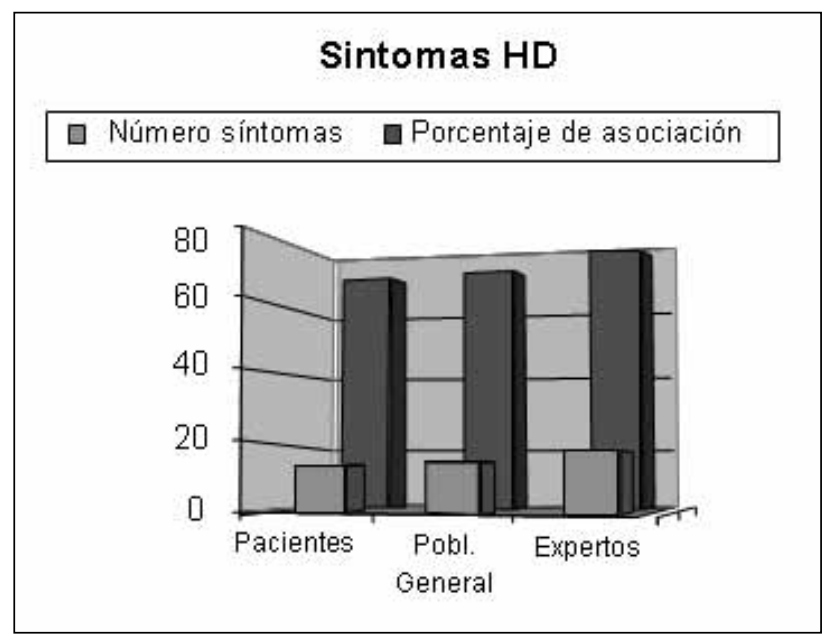

Gráfico 6. Número de síntomas que asocia cada grupo a la HD. Y porcentaje de asociación.

Los participantes valoraron su nivel de acuerdo con los ítems ofrecidos utilizando una escala igual a la empleada en el apartado 1 (escala de cinco puntos donde $1=$ muy en desacuerdo y 5 muy de acuerdo). El gráfico 7 ofrece los resultados del nivel de acuerdo de los distintos grupos en su valoración del factor causal de la IRC. Los hallazgos nos permiten afirmar que para todos los

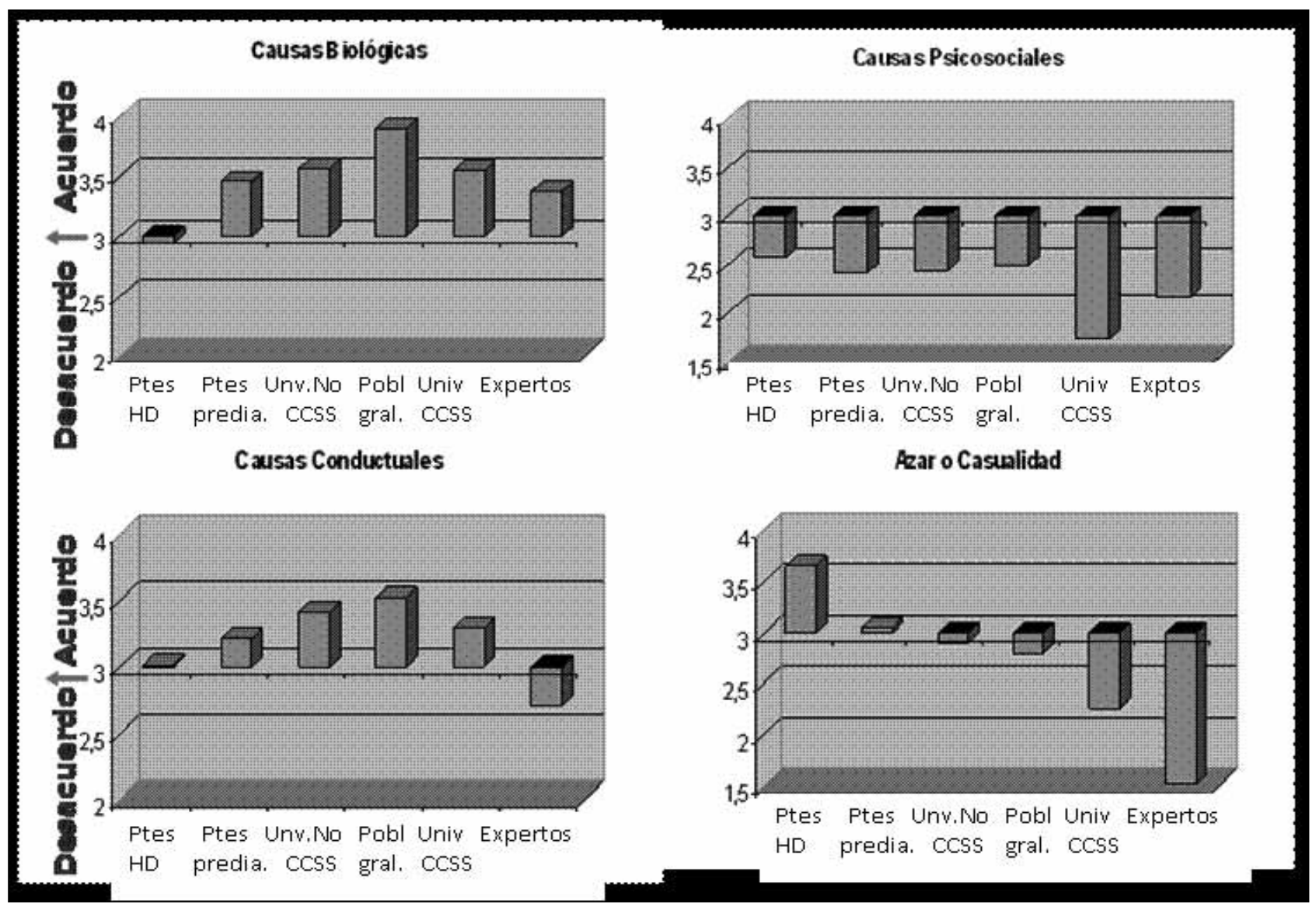

Gráfico 7. 
grupos las causas de la enfermedad son principalmente biológicas, seguidas muy de cerca por las conductuales. Ningún grupo alcanza el acuerdo en la causalidad psicosocial de la IRC. En la categoría Mala suerte, el grupo de pacientes es el que mayores puntuaciones obtiene, con una tendencia clara a considerar el azar factor causal de la IRC.

En cuanto al número de causas que asigna cada grupo a la IRC (gráfico 8), pacientes y expertos nefrológicos, con una mayor experiencia con la IRC, son los que menos asignan, y los que mayor número asocian son la población general.

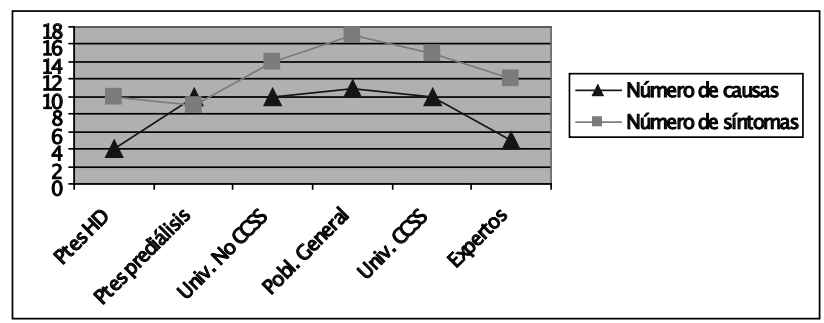

Gráfica 8. Número de causas y síntomas que asocian a la IRC los distintos grupos.

\section{Discusión}

Una posible explicación a las discrepancias encontradas respecto a la curabilidad entre aquellos con conocimientos científico específico de la enfermedad y pacientes (tanto en HD como en prediálisis) podría radicar en el concepto de cura que manejan profanos y científicos. Para los profanos, con experiencia en la enfermedad o sin ella, el trasplante de riñón supone la cura de la enfermedad; en consonancia con otros autores, este concepto parte de la metáfora cuerpo-máquina. Muchos conceptos contemporáneos de la estructura y función del cuerpo se toman prestados de la tecnología, y las máquinas ofrecen los modelos de los cuales la gente conceptualiza y explica la estructura y funcionamiento del cuerpo. Este concepto cuerpo-máquina facilita el entendimiento de que las partes del cuerpo pueden fallar e incluso necesitar ser reemplazados por las piezas de repuesto. La cirugía de trasplante, el uso de marcapasos, distintos tipos de implantes refuerzan la idea del cuerpo como una máquina reparables ${ }^{15,16}$. Siguiendo este modelo, si se cambia el órgano enfermo por uno sano cambiamos enfermedad por salud, terminamos el problema. El trasplante es interpretado como un hecho simple que se describe en función de los cambios observables, mediante una relación causa-efecto simple (realismo ingenuo), característica de las concepciones intuitiva, denominadas también concepciones caseras 0 teorías profanas en la literatura. Pero desde la perspectiva científica, el trasplante es un fenómeno mucho más complejo que se interpreta como un sistema de relaciones de interacción (característica de las concepciones 0 teorías científicas), y supone que el paciente trasplantado tiene que seguir cuidándose porque de modo alguno puede afirmarse que la enfermedad que ocasionara el fracaso del riñón propio haya desaparecido con el recambio del órgano, y que el éxito del trasplante viene también determinado por la integración del nuevo órgano en el organismo receptor; este concepto de trasplante, desde el punto de vista de la ciencia, implica también su interpretación en términos de interacción.

Además, es posible que ante la posibilidad de cura algunos pacientes hayan contestado refiriéndose a las consecuencias de la enfermedad, tratables o controlables con la HD y el trasplante y otros hayan contestado en referencia a la enfermedad en sí, que no tiene cura.

Los resultados obtenidos en el análisis del factor incapacidad sugiere que estudiantes y población general han asumido el modelo biomédico dominante de nuestra cultura, según el cual todas las enfermedades con una cusa orgánica se manifiestan con síntomas físicos, siendo el dolor un síntoma prototípico de enfermedad ${ }^{17,18}$. La experiencia con la enfermedad marca la diferencia. Podríamos aventurar que aquellos que la sufren se adapten a todas las inconveniencias y a la toma de analgésicos como algo normal e implícito al hecho de padecer la enfermedad. En cuanto al criterio de los expertos, son varios los autores que coinciden en la idea de que los profesionales sanitarios en general y las enfermeras en particular valoran el dolor de forma muy distinta si éste es manifestado por un paciente agudo o por uno crónico ${ }^{18,19,20,21}$. En general consideran que el pacientes crónico "sufre" menos dolor (su dolor es menos intenso) que el paciente agudo.

Coincidiendo con los hallazgos de otros autores $22,23,24$, podría ser que la falta de conocimiento específico sobre la enfermedad lleve a buscar una causa para cada uno de los síntomas asociados a la enfermedad. El grupo de población general es el que más causas y más síntomas asocia a la IRC.

\section{Conclusiones}

- En todos los ítems del cuestionario, el grupo de pacientes tiene creencias más cercanas a las mantenidas por novatos que a las de los expertos. 
- Pacientes y novatos consideran que la IRC tiene mayores posibilidades de cura y se produce por causas relacionadas con la conducta o el azar. En el análisis del factor cura, es posible que algunos participantes hayan respondido en referencia a las secuelas o consecuencias de la enfermedad (que en cierta medida si pueden ser controladas por la HD y otras intervenciones terapéuticas) y también es posible que muchos participantes vean la curabilidad de la enfermedad en el trasplante. Una alternativa más de tratamiento, desde el punto de vista de la ciencia, pero en ningún caso una cura.

- Pacientes y expertos coinciden en dos ideas: Ia IRC es menos dolorosa de lo que opinan novatos y menos grave de lo que opinan universitarios de CCSS. Respecto al dolor asociado a la IRC, todos los grupos, salvo aquellos con una experiencia directa con la IRC, han asumido el modelo biomédico hegemónico según el cual todas las enfermedades de causa orgánica se manifiestan con síntomas físicos, siendo el dolor un síntoma prototípico de enfermedad

- Los expertos nefrológicos y universitarios CCSS asocian mayor número de síntomas a la IRC y a la HD. En consonancia con los hallazgos de otros autores, la percepción y calidad de vida de los pacientes analizada por los profesionales que les atienden es peor que la declarada por los propios pacientes.

\section{Limitaciones e implicaciones para la práctica}

El tamaño de la muestra, la naturaleza cambiante de las representaciones en función del conocimiento, cercanía y experiencia con la enfermedad y la evidencia empírica presentada no resultan suficientes para la delimitación de las representaciones de la enfermedad. La profundización en este tema sugiere el uso de diseños longitudinales y métodos de análisis múltiple, no desarrollados en este trabajo.

Las creencias que mantiene las personas sobre la enfermedad crónica son importantes a la hora de comprender las acciones que aquellas tomarán respecto a la enfermedad y su tratamiento, por lo que la práctica enfermera debe incluir una valoración integral de las representaciones de la enfermedad del paciente. Las intervenciones enfermeras que consideran las creencias de los pacientes sobre su situación tienen mayor posibilidad de poder alterar la percepción general de la enfermedad y mejorar los resultados de aquellos con una enfermedad crónica.
Recibido: 27 Agosto 2013

Revisado: 28 Agosto 2013

Modificado: 30 Agosto 2013

Aceptado: 2 septiembre 2013

\section{Bibliografía}

1. Hagger, M. y Orbell, S. A meta-analytic review of the common-sense model of illness representations. Psychology and Health. 2003; 18(2): 141-184.

2. Leventhal, H., Diefenbach, M., Y Leventhal, E. A. Illness cognition: Using common sense to understand treatment adherence and affect cognition treatment. Cognitive Therapy and Research: 1992; 16(2): 143-163.

3. Ferguson $E$, Lawrence $C$, Bibby $P$, Leaviss J, Moghaddam N. Lay Conceptualizations of Occupational Disease. Research Report 469 Prepared by Risk Analysis, Social Processes \& Health (RASPH) Group. 2006. [en linea] [fecha de acceso 2 abril de 2010]. Disponible en: http://www.hse. gov.uk/research/rrpdf/rr469.pdf.

4. Barrowclough C, Lobban F, Hatton C, Quinn J. An investigation of models of illness in carers of schizophrenia patients using the Illness Perception Questionnaire. Br J Clin Psychol. 2001 Nov;40(Pt 4):371-85.

5. Heijmans, M., De Ridder, D., \& Bensing, J. Dissimilarity in patient's and spouse's representations of chronic illness: exploring the relationship with patient's adaptation. Psychology and Health.1999;14: 451-466.

6. Ferguson, E., Farrell, K., Lowe, K. C., \& James, V. Current perceived risks of blood transfusion: The roles of stakeholder knowledge and perceptions. Transfusion Medicine. 2001; 11: 129135.

7. Leventhal $H$, Meyer D, Nerenz D. The common sense representations of Illness danger En Rachman S.(Ed.) Contributions to medical psychology (vol. II). Oxford: Pergamon Press; 1980. Vol 2, p. 2-30. 
8. Leventhal H, Nerenz D, Steele DJ, (1984). Illness Representations and Coping with Health Threats. En Baun A, Taylor SE, Singer JE.(Eds). Handbook of Psychology and Health. New Jersey: Lawrence Erlbaum; 1984.Vol 4. p. 219-252.

9. Bishop GD. Understanding the understanding of illness: lay disease representations. In: J A Skelton and R T Croyle (Eds). Mental representation in health and illness. York: Springer-Verlag; 1991. p87-105.

10. Polaschek N. The experience of living on dialysis: a literature review. Nephrology Nursing Journal. 2003; 30(3): 303-9, 313.

11. Parfeni M, Nistor I, Covic A. Nephrol A systematic reviwe regardin the association of illness perception and survival among end-stage renal disease patients. Dial Transplant. 2013 jun 4. [Epub ahead of print].

12. Lin $\mathrm{CC}$, Chen $\mathrm{Mc}$, Hsieh HF, Chang SC. Illness reptresentations and coping processes of Taiwanese patiens with early-stage chronic kidney disease. J Nurs Res. 2013 Jun; 21(2): 120-8.

13. Vélez $E$, Ramasco M. Meaning of Illness and IIIness Representation, Crucial Factors to Integral Care. EDTNA-ERCA Journal. 2006; 32 (2):81-85.

14. Van-Der Hosfstadt CJ, Rodríguez Marín J. (1997). Adaptación de un cuestionario para la medida de la representación de la enfermedad (Adaptation of a questionnaire to assess illness representation). Psicothema. 1997; 9: 237-245.

15. Sanner MA. Exchanging spare parts or becoming a new person? People's attitudes toward receiving and donating organs, Social Science \& Medicine. 2001;52(10):1491-1499.
16. Helman, C. G. Culture, health and illness. ( $p p$ 2-36). 0xford: Butterworth \& Heinemann; 1994.

17. Engels $G$. The need for a new medical model: A challenge for biomedicine. Science, 1977;129-136.

18. Skelton JA. Laypersons' Judgments of Patient Credibliy and the Study of Illness Representations. En: J A Skelton and R T Croyle (Eds), Mental representation in health and illness. New York: Springer-Verlag. 1991 (pp. 108-131).

19. McCaffery, M., Ferrell, R., Pasero, C. Nurses' personal opinions about patients' pain and their effect on recorded assessments and titration of opioid doses. Pain Management Nursing. 2000; 1 (3): 79-87.

20. Kremenchutzky M, Walt L. Perceptions of health status in multiple sclerosis patients and their doctors. Can J Neurol Sci. 2013 Mar; 40(2):210-8.

21. Green $C R$, Anderson $K 0$, Baker $T A$, Campbell LC, Decker S, Fillingim RB, Kalauokalani DA, Lasch KE, Myers C, Tait RC, Todd KH, Vallerand AH. The unequal burden of pain: confronting racial and ethnic disparities in pain. Pain Med. 2003 Sep;4(3):277-94.

22. Bishop, G.D. Lay conceptions of physical symptoms. Journal of Applied Social Psychology.1987; 17:127-146.

23. French DP, Marteau TM, Weinman J, Senior V. Explaining differences in causal attributions of patient and non-patient samples, Psychology, Health \& Medicine. 2004; 9(3): 259.

24. Murphy EL, Murtagh FE, Carey I, Sheerin NS. Understanding symptoms in patients with advanced chronic kidney disease managed without dialysis: use of a short patient-completed assessment tool. Nephron Clin Pract. 2009; 111(1):c74-80. 